\title{
Основные принципы создания систем автоматизации проектирования и управления в машиностроительных производственных системах
}

Г.Б. Бурдо 1, д.т.н., профрессор, зав. кафедрой "Технология и автоматизаиия машиностроения", gbtms@yandex.ru

Н.А. Семенов ", д.т.н., профессор кафедры "Инфрормаиионные системы"

1 Тверской государственный технический университет, г. Тверь, 170026, Россия

Современное наукоемкое машиностроение и приборостроение относятся к высокотехнологичной сфере производства и имеют свои особенности, которые предопределяют специфику происходящих в них процессов. Поэтому исследование этих особенностей в настоящее время достаточно актуально и востребовано.

В данной статье показаны результаты работы, направленной на выявление процессов, выполняемых в производственных системах, на их автоматизацию и построение эффективных алгоритмов принятия решений.

Дана классификация видов процессов производственных систем машиностроения, определены критерии оценки эффективности. Указанные процессы были исследованы как выполняемые в рамках жизненного цикла продукции на этапах конструирования и изготовления изделий надлежащего качества. Рассмотрены шесть направлений совершенствования процедур принятия решений при выполнении процессов в производственной системе.

Было установлено, что принятие решений должно основываться на определенных критериях их оценки. Основополагающим критерием, по мнению авторов, должно быть качество изделия, определяющее конкурентоспособность продукции в определенном ценовом диапазоне.

Качество и сроки изготовления продукции определяются не только совершенством процессов, но и структурой технологических подразделений производственной системы, поэтому рассмотрены критерии, оценивающие состав этих подразделений.

Для интеграции процессов, выполняемых в производственных системах, использован системный подход, алгоритмы выработки решений реализуются с использованием аппарата искусственного интеллекта.

Ключевые слова: наукоемкое машиностроение, жизненный иикл изделия, проектирование, управление технологическими подразделениями, принятие решений, управление процессами, качество изделия, производственная система.

Известно, что современное наукоемкое машиностроение и приборостроение относятся к высокотехнологичной сфере производства и обладают рядом особенностей, обусловленных наиболее общими причинами. Среди них экстремальные условия эксплуатации изделий, сложность конструкции механики и электроники изделий; отсутствие объективных критериев оценки качества изделий; желание заказчиков иметь изделия, адаптированные под конкретные условия эксплуатации, и отсутствие в связи с этим возможности серийного производства изделия. Кроме того, оказывают влияние и сжатые сроки на разработку и изготовление изделий, что предопределяет необходимость автоматизации всех этапов их жизненного изикла (ЖЦ), потребность в выполнении определенного объема поисковых и опытно-конструкторских работ непосредственно в процессе изготовления изделий, а также необходимость реализации при этом новых технологий и технологических приемов.

Все эти факторы, к сожалению, приводят к большим временным и денежным затратам при реализации проектов по созданию новых изделий, не всегда обеспечивая необходимое качество.

Таким образом, разработка подходов к созданию высокоэффективных комплексно-автоматизированных производственных систем в области машиностроения и приборостроения (далее - машиностроение) востребована временем.

\section{Производственная система и критерии принятия решений в ней}

Производственная система для задач машиностроения - это конструкторские и технологические службы, производственные мощности, испытательные, научные, а также управляющие (в том числе и самой производственной системой) подразделения. В рамках производственной системы выполняются конструкторская подготовка производства (включая научные разработки), технологическая подготовка производства, изготовление и испытание изделий.

Для эффективного решения задач машиностроения необходимо иметь автоматизированные системы проектирования различного назначения (САПР, АСУТП), способные оперативно реагировать на изменения внешней ситуации, организовать итерационные процедуры по выработке приемлемого решения с представителями различных служб, то есть реализовать функциональный способ управления организацией. Очевидно, что основные функции предприятия, связанные с выпуском изделий, реализуются именно в производственной системе, которую в этом случае можно охарактеризовать как организационно-технологическую.

Рассмотрим критерии, в соответствии с которыми в автоматизированных системах современной производственной системы должны приниматься решения. К сожалению, в последнее время акцент в производ- 
ственных организационно-технологических системах сместился в сторону экономики, извлечения максимальной прибыли (причем вопреки здравому смыслу) и не вполне учитываются требования конкурентоспособности изделий. В долгосрочной перспективе такой подход для предприятия неэффективен.

Определяющей целью машиностроения должен быть выпуск конкурентоспособной продукции. Конкурентоспособность достигается обеспечением требуемого (заказчиком, тенденцией развития отрасли) качества изделий, их выпуском в заданные сроки и при заданном уровне производственных затрат (временных и денежных). В таком ракурсе управление качеством становится не довеском к какой-либо системе управления организацией, а основополагающим процессом. Весьма эффективным инструментарием такого управления могут быть автоматизированные системы.

Таким образом, целью управления (и автоматизированного) производственными системами должно быть достижение качества при одновременном сокращении временных и материальных затрат.

\section{ЖЦ изделия и автоматизация управления процессами в производственных системах}

Анализ ЖЦ продукции составляет методологическую основу $[1,2]$ анализа процессов деятельности организации, так как представляет собой инструмент управления сведениями об изделии, его состоянии, уровне качества. ЖЦ изделия охватывает период от начала его создания до утилизации.

В наукоемком машиностроении предприятия, как правило, одновременно являются и разработчиками, и изготовителями своих изделий, а эксплуатация, ремонт и утилизация выполняются потребителями.

Для современных производственных систем, в частности машиностроения, главное - это управление качеством, поэтому, выполняя этапы ЖЦ, их продолжение либо завершение, необходимо определять структуру и функции процессов, задействованных в проектах по созданию изделий, исходя из достижения предполагаемого уровня [3].

Представим модель процессов обеспечения качества (см. рисунок), отвечающую случаю, когда обеспечение необходимого уровня качества является целью работы производственной системы и управление выполняется с использованием автоматизированных систем. На рисунке использованы следующие обозначения: АСУП - АСУ организацией (предприятием); АСУ ТППиИ - АСУ технической подготовкой производства, изготовления и испытания опытного изделия, то есть на этапах ЖЦ изделия; $A$ - множество критериев показателей качества изделия и (или) качества выполнения работ на этапах; $D_{0}$ - блок задания, формирующий множество технических заданий (Т3); $T_{1}-$ ТЗ на проведение НИОКР; $T_{2}$ - ТЗ на проведение конструкторской подготовки производства (КПП); $T_{3}-$

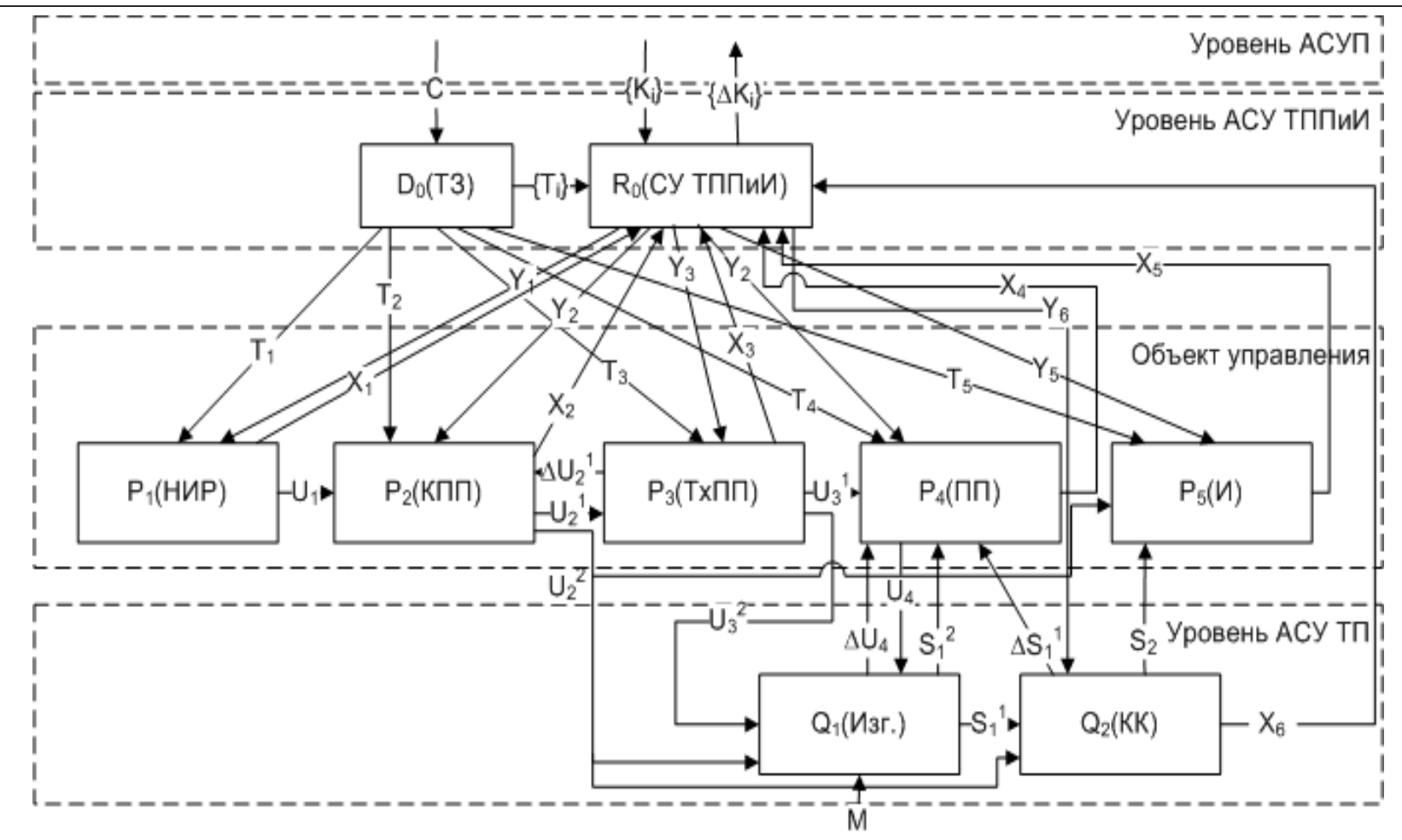

Модель автоматизированных прочессов обеспечения качества

A model of automated quality maintenance processes 
Т3 на проведение технологической подготовки производства (ТхПП); $T_{4}$ - ТЗ на проведение планирования производства (ПП) по реализации технологических процессов в производственной системе; $T_{5}-$ ТЗ на проведение испытаний изделия; $R_{0}$ - оператор, управляющий работой на всех этапах создания образца наукоемкой продукции; $R_{0}^{1}$ - управление оператором $P_{1}$ (НИОКР); $R_{0}^{2}$ - управление оператором $P_{2}$ (КПП); $R_{0}^{3}$ - управление оператором $P_{3}$ (ТхПП); $R_{0}^{4}-$ управление оператором $P_{4}$ (ПП); $R_{0}^{5}$ - управление оператором $P_{5}$ (испытания изделия, И); $R_{0}^{6}$ - управление работой оператора $Q_{2}$ (контроль качества (КК) работ); $R_{0}^{7}$ - оператор по координация управления с АСУП; $U_{1}-$ множество параметров, содержащихся в отчетной документации по НИОКР, необходимых для проведения КПП; $U_{2}^{1}-$ множество параметров, содержащихся в конструкторской документации и необходимых для ТхПП; $\Delta U_{2}^{1}-$ множество параметров, определяющих требования оператора $P_{4}$ по повышению уровня технологичности изделия; $U_{2}^{2}-$ множество параметров, содержащихся в конструкторской документации и необходимых для изготовления изделий в технологической системе, контроля и испытаний; $U_{3}^{1}$ - множество параметров технологических процессов по трудозатратам и станкозатратам (трудозатратам); $Q_{1}$ - оператор, выполняющий изготовление изделий (производственная система); $U_{3}^{2}$ - множество параметров, определяющих реализацию технологических процессов на рабочих местах (технологические процессы, инструкции, управляющие программы для станков с ЧПУ); $S_{1}^{2}$ множество параметров, характеризующих состояние производственной системы (загрузка рабочих мест, заказы в исполнении, ход выполнения планов и т.д.); $U_{4}-$ множество параметров, характеризующих планы различных уровней по выпуску образца продукции; $\Delta S_{1}^{1}$ - множество параметров - сведения об изделиях, не прошедших контроль и нуждающихся в доработке (переделке); $\Delta U_{4}-$ множество параметров, характеризующих диспетчирование работ по данному изделию; $S_{2}$ - материальная связь - изделие (образец продукции); $Q_{1}$ - оператор изготовления опытного образца изделия; $M$ - материалы и покупные изделия; $S_{1}^{1}-$ изготовленные образцы продукции и сведения об их готовности; $Q_{2}$ - оператор контроля деталей, узлов и изделий целиком.

Модель может быть использована для выявления и описания функциональных процессов управления в следующей последовательности.

В первую очередь следует выявить, какие действия, выполняемые на протяжении ЖЦ изделия в рассматриваемой производственной системе, влияют на качество предполагаемого к выпуску изделия (обозначим их как действия группы 1). На рисунке это этапы ЖЦ изделия: научно-исследовательская работа, конструкторская подготовка производства, технологическая подготовка производства, изготовление (с планированием, как управление изготовлением и контролем) и испытания. Понятно, что этими процессами надо управлять [4], появляется верхний уровень управления (определение ТЗ и собственно управление этапами ЖЦ изделия). Реализация технологических процессов идет в производственных подразделениях, поэтому появляется низший уровень - уровень АСУ ТП. Таким образом, процессы первой группы реализуются на четырех уровнях. Первый уровень - АСУП, координация всех работ в рамках предприятия. Второй уровень - управление качеством на этапах ЖЦ изделия. Третий уровень - реализация процессов на этапах ЖЦ изделия. Четвертый уровень - управление технологическими процессами. Процессы на этих четырех уровнях являются базовыми для предприятия.

Процессы группы 1 не могут существовать сами по себе, поэтому следует выявить действия (группы 2), непосредственно не влияющие на качество изделия, но необходимые для выполнения действий группы 1 , например, транспортные работы, документооборот, управление финансами, заключение договоров и контрактов и т.д. Следовательно, совокупность этих действий и будет составлять суть процессов, реализуемых на предприятии. Далее необходимо будет продетализировать процессы, разбив их на операции. Каждая операция должна иметь законченный функциональный смысл, включать выполнение одной функции в рамках процесса. Также важно найти «золотую середину» между концентрацией и дифференциацией действий в операции, исходя из квалификации исполнителей и возможности принятия ими самостоятельного решения. Далее определяют контрольные места в процессе, в которых выполняется оценка качества.

Затем следует привязать процессы группы 2 к процессам группы 1 по времени. Увязку по времени процессов группы 1 следует выполнять между собой.

Немаловажным является и определение ответственных за процессы на каждом уровне, так как в их числе участники из разных (по административной структуре) подразделений. Сложность в том, что участвующие в процессе подразделения организации уже имеют своих руководителей. Как правило, руководить процессом должен наиболее грамотный (досконально понимающий суть процесса) из руководителей участвующих подразделений. Задача руководителей других подразделений - четко распределить работу по исполнителям. Здесь получается логичное, но, к сожалению, не всегда принимаемое распределение руководства по функциональному (не административному) признаку. Руководитель процесса низшего уровня функционально подчиняется руководителю процесса, в который он входит. 


\section{Критерии оценки качества решений на этапах ЖЦ изделия}

Контроль качества решений, направленных на обеспечение качества изделия, будет выполняться между операциями процесса. Чтобы были понятны действия при оценке решений, следует сопоставить операции процесса этапам ЖЦ изделия, которые определяют общепринятый смысл решений (НИР, КПП, ТхПП, изготовление (включая управление технологическими процессами), испытания).

Критерии качества любого процесса деятельности организации могут не только определяться моделью качества изделия, но и дополняться критериями качества самого процесса.

Так, процессы КПП зачастую имеют непосредственную связь с параметрами модели качества изделия (например, производительность, мощность, коэффициент унификации и т.д.). А, скажем, процессы ТхПП влияют опосредованно. Они должны обеспечить разработку технологических процессов изготовления изделия в соответствии с конструкторской документацией.

В процессах приобретения сырья (этап «изготовление» ЖЦ изделия) немаловажна возможность точного указания, какое именно сырье, с какими параметрами и у какого поставщика надо приобрести (дополняемые критерии качества). Обязательный дополнительный критерий процесса - время исполнения функций отдельных операций, входящих в процесс.

Учитывая, что многие оценки будут носить неявный качественный характер, важное значение приобретает подбор экспертов из числа работников организации для оценки решений.

\section{Совершенствование процедур принятия решений при выполнении процессов в производственной системе}

Как уже отмечалось, одним из важнейших параметров, определяющих эффективность работы производственных систем, являются временные затраты на выполнение этапов ЖЦ изделия, реализуемых в рамках данного предприятия. Рассмотрим мероприятия, позволяющие сократить время выпуска образца нового изделия.

1. Автоматизация процедур управления процессами с точки зрения их иерархии и временного фактора. Должна быть реализована в виде системы с сервис-ориентированной архитектурой, с определенными правами доступа каждого пользователя.

2. Автоматизация инженерных работ при выполнении процессов, связанных с КПП, ТхПП, изготовлением и испытаниями. Особенность для условий рассматриваемых производственных систем в мобильности и в возможности с их помощью оперативно выполнять корректировку решений. Для этого про- граммные средства на уровне пользователей должны быть информационно интегрированы между собой (САПР КПП с САПР ТхПП, АСУТП с САПР ТхПП, АСУТП с производственными подразделениями), а на верхнем уровне интегрированы в АСУП. В этом случае появляется возможность оперативно корректировать решения (например, конструкторские на основе выявленных на этапе ТхПП недочетов), проектировать технологию на основе знания текущей производственной ситуации и т.п.

3. Параллельное выполнение работ, включенных в разные этапы ЖЦ изделия (или в разные стадии одного этапа). Понятно, что процессы конструкторской подготовки производства начинают проект по созданию нового изделия. Но уже на стадии, соответствующей эскизному проекту (так как в рассматриваемых типах производственных систем стадийность КПП, как правило, не выдерживается), можно выполнять и технологическую подготовку производства: анализ технологичности конструкции, предварительную проработку технологии, подбор средств технологического оснащения и их заказ (при необходимости). Также может быть выполнен заказ и дефицитных позиций (или с большим периодом доставки) материалов и комплектующих. Унифицированные детали и узлы, предполагаемые к использованию в новом изделии, могут быть запущены в изготовление. Таких примеров можно привести множество.

Однако способ реализации данного мероприятия достаточно прост. С этой целью необходимо, учитывая специфику конкретного предприятия, сделать соответствующим образом временную увязку процессов, входящих в разные этапы ЖЦ изделия (или в разные стадии одного этапа), и приглашать для обсуждения технических решений работников, ответственных за соответствующие операции процессов, включая и процессы 2-й группы.

4. Разработка интеллектуальных систем поддержки принятия решений при реализации процессов. Прежде всего речь идет о решениях, принимаемых и реализуемых представителями различных функциональных подразделений. Системы поддержки решений строятся на основе экспертных оценок и позволяют избегать тупиковых ситуаций. Еще одно их достоинство решение может обсуждаться и приниматься дистанционно.

5. Создание предпосылок для эффективного принятия решений на последующих этапах и стадиях ЖЦ изделия.

Унификация деталей и их элементов упрощает не только проектирование изделий, но и технологическую подготовку производства за счет применения унифицированных технологических решений. Закрепление операций обработки за наименее загруженным оборудованием (проектирование на основе текущей производственной ситуации) уменьшает вероятность появления узких мест и упрощает управление технологическими процессами. 
6. Построение всех процессов организации (не только производственных) в соответствии с идеологией бережливого производства: делать то, что надо именно сейчас, и уменьшать непроизводительные затраты труда и средств. Наиболее важно это требование для процессов организации, реализуемых высшим управленческим звеном [5].

\section{Основные требования к технологическим подразделениям производственной системы}

Очевидно, что совершенство производственных (технологических) подразделений во многом определяет и эффективность работы всей производственной системы. Специфика многономенклатурного наукоемкого производства вносит определенную специфику в перечень требований, реализуемых при создании технологических подразделений. Рассмотрим, на взгляд авторов, наиболее важные из них.

1. Обеспечение резервов производственных мошноcmeй. Как показывает опыт, оборудование каждой группы должно быть загружено плановыми работами не более, чем на 80 \%. В этом случае уменьшается (или исключается) время ожидания следующей операции. Всегда есть возможность выполнения срочных внеплановых работ, демпфирования непредвиденных ситуаций.

2. Пропоричиональность производственных мощчностей по видам оборудования. Пропорциональность мощностей достигается на основе структуры станкоемкостей (трудоемкостей) предполагаемых к выпуску изделий. Таким образом, можно говорить о «штучной» пропорциональности мощностей.

3. Производственная гибкость. Оборудование должно иметь широкие технологические возможности в пределах типов оборудования не только одной группы, но и разных групп. Скажем, обрабатывающие центры вертикальной компоновки вертикально и радиально перекрывают технологические возможности сверлильной, вертикально - фрезерной, вертикальной координатно - расточной групп оборудования (понятно, что в рамках соответствующих размерных групп). Такой подход обеспечивает возможность выполнения операции на нескольких рабочих местах, то есть производственную гибкость.

4. Технологическая гибкость оборудования. Под параметром, характеризующим технологическую гибкость, понимается время переналадки оборудования на выполнение другой операции. Данный параметр определяет технико-экономические показатели эксплуатации оборудования. Чем больше времени станок находится в переналадке (a, следовательно, непосредственно не занят в изготовлении детали), тем ниже эффективность его работы.

В этом смысле весьма перспективно использование оборудования с ЧПУ. Однако его эффективное использование во многом определяется уровнем техно- логической и организационной подготовки производства. Если с технологической подготовкой (ее технической составляющей) в целом все понятно (есть различные программные реализации, хороший рынок инструментального обеспечения и т.д.), то прогрессивные способы ее ведения, установление очередности выполнения операций для различных партий деталей на станках с ЧПУ - инженерная многовариантная задача, которая должна решаться на основе минимизации суммарного времени переналадок для всех рассматриваемых партий деталей, если нет жестких приоритетов.

Унификация технологических решений (схем наладок, элементов управляющих программ и т.д.) также имеет своей целью сокращение простоя оборудования под наладкой.

Немаловажный фактор - организация наладки оборудования. Как правило, для станков с ЧПУ применяется многостаночное обслуживание, поэтому могут быть простои оборудования (ожидание переналадки) вследствие занятости рабочего на другом станке. В этой ситуации, в принципе, возможна наладка специальным персоналом за счет увеличения численности рабочих или организации бригадной работы без увеличения численности (взаимопомощь). Таким образом, технологическая гибкость - комплексное понятие, охватывающее разные аспекты (технический, технологический, организационный).

5. Расстановка оборудования. От рациональности расстановки оборудования в производственных подразделениях многономенклатурных производств напрямую не зависит время технологических циклов, но создаются предпосылки для негативного влияния на него. Нерациональная расстановка приводит к увеличению длины межоперационных перемещений партий деталей, поэтому увеличатся и временные затраты для их осуществления. Если эту работу выполняют основные рабочие, то у них остается меньше времени на производительную работу. Если перемещение деталей между операциями выполняют вспомогательные рабочие, то потребуется увеличение их численности.

Указанные предложения облегчают и упрощают разработку алгоритмов управления технологическими подразделениями.

\section{Требования к кадрам и кадровой политике}

Bce предложенные мероприятия невозможно успешно реализовать без соответствующего кадрового обеспечения. Рассмотрим основные моменты.

1. Выработка у исполнителей чувства взаимопомощи и коллективной ответственности за результат. Следует понимать, что разделение функций в рамках общего процесса не означает разделение коллектива, все действия должны быть направлены на общий успех - выпуск качественного изделия. Здесь, как показывает опыт, не обойтись без создания нужной кор- 
поративной культуры и использования методов стимулирования деятельности работников (именно стимулов, а не антистимулов).

2. Повышение квалификации исполнителей должно вестись постоянно как силами работников самой фирмы (курсы, самообучение), так и во внешних организациях (семинары, обмен опытом и т.п.). Весьма важна кадровая подготовка цехового управленческого персонала, особенно мастеров и руководителей диспетчерских служб. Задача - умение вырабатывать решения по управлению технологическими циклами и производственными мощностями, так как на практике нет подходящих для этих целей (для многономенклатурных производств) цеховых управленческих автоматизированных систем класса Scada.

Важно и обучение работников умению заменять друг друга в рамках смежных функций.

3. Немаловажны готовность руководства организации на серьезные технические и кадровые инновации, компетентность в принятии управленческих решений.

Материальные ресурсы на выполнение описанных мероприятий нужны не единовременно. Для условий конкретного предприятия всегда можно составить гибкий проект. Но надо понимать, что качественное изделие не бывает дешевым, в том числе и в силу больших затрат на подготовительном этапе.

\section{Заключение}

Данная работа носит во многом принципиальный характер, а порой, возможно, и спорный. Цель ее определение ключевых принципов, в соответствии с которыми, на взгляд авторов, должны развиваться современные системы автоматизации процессов выработки и принятия решений в машиностроительных производственных системах [6-8]. Специфичность условий, возможностей, видов изготавливаемых изделий предполагает и особенности подходов при реализации мероприятий. Однако в любом случае уже сам факт исследования возможности внедрения мероприятий заставляет серьезно анализировать работу и состояние предприятия, помогает увидеть и устранить недостатки. Для управления процессами деятельности предприятия и принятия решений целесообразно применять методы теории систем, искусственного интеллекта и нечетких множеств [9-11].

Настоящая работа находится на стадии апробации алгоритмов управления процессами обеспечения качества изделия на ряде машиностроительных и приборостроительных предприятий.

Работа выполнена при поддержке РФФИ, проект № 17-01-00566.

\section{Литература}

1. Колчин А.Ф., Овсянников М.В., Стрекалов А.Ф., Сумароков С.В. Управление жизненным циклом продукции. М.: Анахарсис, 2002. $304 \mathrm{c}$.

2. Никифоров А.Д., Бакиев А.В. Процессы жизненного цикла продукции в машиностроении. М.: Абрис, 2011. 688 с.

3. Бурдо Г.Б., Сорокин А.Ю. Автоматизация процессов управления качеством при создании наукоемких геофизических изделий // Каротажник. 2016. № 9. С. 185-199.

4. Бурдо Г.Б. Совершенствование технологической подготовки машиностроительного производства // Станки и инструмент (СТИН). 2016. № 7. С. 2-8.

5. Вумек Джеймс П., Джонс Даниел Т. Бережливое производство. Как избавиться от потерь и добиться процветания вашей компании. М.: Альпина Бизнес Букс, 2008. 473 с.

6. Бурдо Г.Б., Стоянова О.В. Автоматизированная система управления процессами создания наукоемких машиностроительных изделий // Программные продукты и системы. 2014. № 2. C. $164-170$

7. Гличев А.В. Основы управления качеством продукции. М.: Стандарты и качество, 2001. $424 \mathrm{c}$.

8. Дитрих Я. Проектирование и конструирование: Системный подход; [пер. с польск.]. М.: Мир, 1981. 456 с.

9. Месарович М., Такахара Я. Общая теория систем: математические основы. М.: Мир, 1978. 311 с.

10. Рыбина Г.В. Основы построения интеллектуальных систем. М.: Финансы и статистика; Инфра-М, 2010. 432 с.

11. Еремеев А.П., Куриленко И.Е. Средства темпорального вывода для интеллектуальных систем реального времени: в кн. Интеллектуальные системы. М.: Физматлит, 2010. Вып 4. С. 222-252.

Software \& Systems

DOI: $10.15827 / 0236-235 X .125 .134-140$

\section{Basic principles of creating design and control automation systems in engineering production systems}

G.B. Burdo ${ }^{1}$, Dr.Sc. (Engineering), Professor, Head of Chair, gbtms@yandex.ru

N.A. Semenov ${ }^{1}$, Dr.Sc. (Engineering), Professor

${ }^{1}$ Tver State Technical University, Tver, 170026, Russian Federation

Abstract. Modern high-tech engineering and instrument making are high-tech production. They have a number of features that determine the specifics of the processes in them. Therefore, the study of these features is quite relevant and in demand nowadays.

The paper presents the results of work aimed at identifying the processes performed in production systems, their automation and construction of effective decision-making algorithms.

There is a classification of the types of processes performed in mechanical engineering production systems, the criteria for evaluating process effectiveness. The abovementioned processes are investigated as the life cycle processes at the stages of product design 
and manufacturing. The authors consider six directions of improving decision-making procedures during process performance in a production system.

It is established that decision-making should be based on their certain evaluation criteria. It is proposed that such fundamental criterion should be product quality, which determines product competitiveness in a certain price range.

Product quality and timing are determined by process improvements, as well as by the structure of production system technological units. Therefore, the paper considers the criteria that evaluate the structure of technological units.

A systematic approach integrates the processes in production systems. Decision-making algorithms are implemented using artificial intelligence.

Keywords: high-tech engineering, product life cycle, design, management of technological units, decision making, process management, product quality, production system.

Acknowledgements. The work has been supported by RFBR, project no. 17-01-00566.

\section{References}

1. Kolchin A.F., Ovsyannikov M.V., Strekalov A.F., Sumarokov S.V. Product Lifecycle Management. Moscow, Anakharsis Publ., 2002, 304 p.

2. Nikiforov A.D., Bakiev A.V. Product Life Cycle Processes in Engineering. Moscow, Abris Publ., 2011, 688 p.

3. Burdo G.B., Sorokin A.Yu. Automation of quality management processes in the development of high-tech geophysical products. Karotazhnik. 2016, no. 9, pp. 185-199 (in Russ.).

4. Burdo G.B. Improving the technological preparations for manufacturing production. Russian Engineering Research. 2017, vol. 37, iss. 1, pp. 49-56.

5. Womack J.P., Jones D.T. Lean Thinking: Banish Waste and Create Wealth in Your Corporation. Free Press, 2003,400 p. (Russ. ed.: Moscow, Alpina Biznes Buks Publ., 2008, 473 p.).

6. Burdo G.B., Stoyanova O.V. An automated process control system for high-tech engineering products. Software \& Systems. 2014, no. 2, pp. 164-170 (in Russ.).

7. Glichev A.V. The Basics of Product Quality Management. Moscow, Standarty i kachestvo Publ., 2001, 424 p.

8. Dietrich Ja. System and Design. Warsaw, 1978 (Russ. ed.: Moscow, Mir Publ., 1981, 456 p.).

9. Mesarovich M., Takakhara Ya. General Systems Theory: Mathematical Foundations. Moscow, Mir Publ., 1978, 311 p.

10. Rybina G.V. The Basics of Building Intelligent Systems. Moscow, Finansy i statistika, Infra-M Publ., 2010, 432 p.

11. Eremeev A.P., Kurilenko I.E. Means of temporal output for intelligent real-time systems. Intellectual Systems. Moscow, Fizmatlit Publ., 2010, iss. 4, pp. 222-252 (in Russ.).

\section{Примеры бибциографического описания статьи}

1. Бурдо Г.Б., Семенов Н.А. Основные принципы создания систем автоматизации проектирования и управления в машиностроительных производственных системах // Программные продукты и системы. 2019. T. 32. № 1. C. 134-140. DOI: 10.15827/0236-235X.125.134-140.

2. Burdo G.B., Semenov N.A. Basic principles of creating design and control automation systems in engineering production systems. Software \& Systems. 2019, vol. 32, no. 1, pp. 134-140 (in Russ.). DOI: 10.15827/0236235X.125.134-140. 